\title{
Coding Schemes for Energy Efficient Multi-Source Cooperation Aided Uplink Transmission
}

\author{
Rong Zhang and Lajos Hanzo
}

\begin{abstract}
In this paper, we consider coding schemes designed for multi-source cooperation, where a cluster of sources cooperatively communicates with the destination. More explicitly, we propose both a powerful superposition coding scheme and a physical-layer algebraic network coding scheme. Our simulation results demonstrate that both of the proposed schemes are capable of performing close to the outage probability bound. Compared to the superposition coding scheme considered, the proposed algebraic network coding arrangement imposes a much lower complexity at the cost of a slight performance degradation, while maintaining the same throughput and delay.
\end{abstract}

Index Terms-Author, please supply your own keywords or send a blank e-mail to keywords@ieee.org to receive a list of suggested keywords.

\section{INTRODUCTION}

$\mathbf{R}$ ECENTLY, the Cooperative Multiple Access (CMA) channel has attracted substantial research interests, where multiple sources forming a cluster of cooperating nodes communicate with the destination, which is also known as Multi-Source Cooperation (MSC) [1], [2]. We proposed in [3] an error-resilient yet high-throughput non-orthogonal interleaved random STC scheme, which was specially contrived for MSC. In contrast to the uncoded system of [3], in this contribution, we aim for improving the energy efficiency of our proposed MSC framework with the aid of two specifically designed coding schemes, namely SuperPosition Coding (SPC) and a Physical-layer Algebraic Network Coding (PANC) scheme.

In contrast to classic time-multiplexing, in the SPC scheme the multiple sources' information is code-multiplexed in order to generate the superimposed and appropriately rotated composite signal, which results in a high throughput. Thus we will introduce an outer channel-coded SPC-aided MSC arrangement, which will be used as the benchmarker of the proposed PANC scheme. On the other hand, the philosophy of Network Coding (NC) was proposed by [4] for the sake of enhancing the wired channel's capacity. Apart from the original

Manuscript received October 21, 2008; revised January 03, 2009. This work has formed part of the Core 4 Research Programme of the Virtual Center of Excellence in Mobile and Personal Communications, Mobile VCE, (www.mobilevce.com), and was supported by EPSRC. Fully detailed technical reports on this research are available to Industrial Members of Mobile VCE. The associate editor coordinating the review of this manuscript and approving it for publication was Prof. Weifeng Su.

The authors are with the School of ECS, University of Southampton, Southampton SO17 1BJ, U.K. (e-mail: 1h@ecs.soton.ac.uk; http://www-mobile.ecs.soton.ac.uk).

Digital Object Identifier 10.1109/LSP.2009.2015996 network-layer applications, it has recently been recognized that the physical-layer of wireless networks also benefits from NC [5]-[7]. However, its extension to MSC is not straightforward [8]. We therefore generalise the concept of network coding and propose the so-called PANC scheme.

In a nutshell, the novel contribution of this paper is that we propose both a SPC scheme and a PANC scheme, which are capable of performing close to the best possible outage probability bound in the context of MSC. Our numerical results show that compared to SPC, the novel PANC arrangement exhibits a reduced complexity $(\iota)$ at the cost of a slight performance degradation $\left(P_{e}^{b l}\right)$, while maintaining the same throughput $(\eta)$ and delay $(\tau)$.

The rest of the paper is organized as follows. In Section II, we describe our MSC model and propose the SPC and PANC schemes considered. Furthermore, the iterative receiver structure and the soft PANC decoding algorithm advocated are also discussed. In Section III, the outage probability bound of MSC is analysed and the numerical results characterizing both schemes are provided. Finally, we conclude our discourse in Section IV.

Notation: Throughout the paper, lower (upper) case boldface letters will represent row vectors (matrices). The identity matrix of size $N$ is denoted as $\mathbf{I}_{N}=\operatorname{diag}[1, \ldots, 1]_{N}$. The superscript $(\cdot)^{T}$ denote transposition. The superscript $(\cdot)^{(1)}$ and $(\cdot)^{(2)} \mathrm{de}-$ notes Phase-I and Phase-II cooperation, while $N_{i}$ and $N_{c}$ represent the information bit duration and codeword length, respectively.

\section{SYSTEM DESCRIPTION}

\section{A. Cooperation Model}

Consider a cluster of single-antenna sources cooperatively communicating with a destination employing a single receive antenna, which jointly result in a Virtual Multiple Input Single Output (VMISO) system. In this VMISO cluster, we assume having a total of $N$ Cooperating Sources (CS), $K$ Active Sources (AS) and $(N-K)$ Relaying Sources (RS). Our MSC scheme entails two phases and is assumed to be symbol-synchronised. In Phase-I cooperation, the source information emanating from all $K$ ASs is broadcast to all $N$ CSs in a Time Division Duplex (TDD) manner ${ }^{1}$. By contrast, Phase-II cooperation is defined as the joint transmission of a combined signal generated by the concerted action of all the $N$ CSs. Therefore, each CS transmits multiple ASs' information, resulting in a high throughput. And each AS is served by multiple CSs and hence benefits from a high diversity gain.

\footnotetext{
${ }^{1}$ The reception of the signal by the destination during Phase-I cooperation provides simply another diversity, which is ignored for simplicity.
} 


\section{B. Cooperative Code Design}

In this paper, we focus our attention on developing coding schemes for MSC in Phase-II cooperation, when the so-called decode-forward relaying technique is employed at each of the $N \mathrm{CSs}$

1) Superposition Coding: Following Phase-I cooperation, the $n$th of the $N$ CSs retrieves all the $K$ ASs' information $\hat{\mathbf{s}}_{k, n}^{(1)}$, $k \in[1, K]$ and the transmitted codeword is constructed as follows. Firstly, the $n$th CS forms $K$ parallel codewords $\mathbf{c}_{n, k}=$ $\pi_{k}\left[f\left(\hat{\mathbf{s}}_{k, n}^{(1)}\right)\right], k \in[1, K]$, where $\pi_{k}$ is referred to as the AS-specific interleaver and $f(\cdot)$ represents the outer channel coding function, which is assumed to be the same for all ASs. These AS-specific outer codewords are then punctured according to $\tilde{c}_{n, k}(i)=c_{n, k}[N(i-1)+n], i \in\left[1, N_{c} / N\right]$. This is followed by a Parallel-to-Serial (P/S) conversion in order to create a single codeword $\mathbf{c}_{n}$. Finally, the composite codeword transmitted from the $n$th CS is BPSK modulated and linearly superimposed, i.e. $x_{n}^{(2)}(i)=\sum_{l=1}^{L_{n}} \rho_{n, l} e^{j \theta_{n, l}} x_{n, l}^{(2)}(i)$, where $x_{n, l}^{(2)}(i)=2 c_{n}\left[L_{n}(i-\right.$ $1)+l]-1, i \in\left[1, N_{c} K / N L_{n}\right]$ and $L_{n}$ is referred to as the number of layers contributed by the $n$th CS's superposition, while $\rho_{n, l}$ subject to $\sum_{l=1}^{L_{n}} \rho_{n, l}^{2}=1$ and $\theta_{n, l} \in[0, \pi)$ denotes the layer-specific amplitude and phase rotation respectively. In this treatise, we assume $L_{n}=L, \rho_{n, l}=\rho_{l}, \theta_{n, l}=\theta_{l}$, $\forall n \in[1, N]$. And for simplicity, equal amplitude allocation and uniform phase rotation are employed.

2) Physical-Layer Algebraic Network Coding: NC may be viewed as a technique of conveying a linear combination of multiple information streams, rather than using conventional routing or relaying for delivering these information streams individually with the aid of classic resource allocation, such as time-multiplexing or code-multiplexing. We generalise the concept of NC as a coding function $f(\cdot)$, which jointly encodes all the incoming multiple information streams. With the aid of this generalisation, the original NC operation $\oplus$ of $K$ linearly coded information streams $\mathbf{s}_{i} \mathbf{G}_{i}, i \in[1, K]$ becomes equivalent to encoding the vectors $\mathbf{s}=\left[\mathbf{s}_{1}, \ldots, \mathbf{s}_{K}\right]$ using a nested Generator Matrix $(\mathrm{GM}) \mathbf{G}=\left[\mathbf{G}_{1}, \ldots, \mathbf{G}_{K}\right]^{\mathrm{T}}$.

We now proceed to describe the construction of codewords for our MSC taking this novel PANC principle into account. After retrieving all the $K$ ASs' information denoted by $\mathbf{s}=$ $\left[\hat{\mathbf{s}}_{1, n}^{(1)}, \ldots, \hat{\mathbf{s}}_{K, n}^{(1)}\right]$ and having a length of $K N_{i}$, the $n$th CS generates a total of $\kappa$ number of versions of the differently interleaved information stream and the resultant codeword $\mathbf{c}_{n}$ of length $N_{c}=\kappa K N_{i}$ is given by

$$
\begin{aligned}
\mathbf{c}_{n} & =\pi_{1}(\mathbf{s}) \mathbf{G}_{1} \oplus, \ldots, \oplus \pi_{\kappa}(\mathbf{s}) \mathbf{G}_{\kappa} \\
& =\left[\pi_{1}(\mathbf{s}), \ldots, \pi_{\kappa}(\mathbf{s})\right]\left[\mathbf{G}_{1}, \ldots, \mathbf{G}_{\kappa}\right]^{\mathrm{T}}
\end{aligned}
$$

where we have $\pi_{i}$ represents $i$ th random interleaver. Although in principle an arbitrary $\mathbf{G}=\left[\mathbf{G}_{1}, \ldots, \mathbf{G}_{\kappa}\right]^{\mathrm{T}}$ may be applicable, we adopt a simple unity-rate ACcumulate Code (ACC), having a GM represented as the upper triangular matrix having entries equal to one. Apart from the non-systematic PANC GM, we may also generate a systematic PANC by designing the GM as

$$
\mathbf{G}=\left[\begin{array}{cc}
\mathbf{I}_{\boldsymbol{\kappa}^{s}} & \mathbf{0} \\
\mathbf{0} & \mathbf{G}_{a c c}
\end{array}\right],
$$
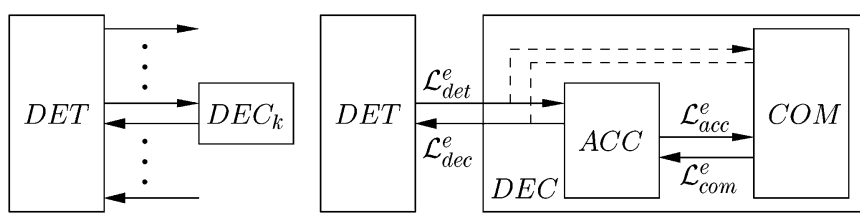

Fig. 1. Iterative receiver architecture of both the SPC scheme employing single-stream decoding (left) and PANC scheme employing multiple-streams decoding (right), where $C O M$ represents the soft combiner of the multiple streams. Systematic and non-systematic segment are denoted as broken and solid line, respectively.

where $\kappa^{s}$ number of differently interleaved versions of the original information streams are created, corresponding to $\left[\pi_{1}(\mathbf{s}), \ldots, \pi_{\kappa^{s}}(\mathbf{s})\right]$. Finally, the $n$th CS transmits a BPSK modulated punctured codeword according to $x_{n}^{(2)}(i)=2 c_{n}[N(i-1)+n]-1, i \in\left[1, N_{c} / N\right]$.

Remarks: The concept of NC and SPC may have some intrinsic links. In fact, the authors of [7] considered the NC concept as a SPC scheme defined over the Galois Field 2 (i.e., the operation + in SPC is replaced by $\oplus$ in terms of NC), while the authors of [8] considered the SPC concept as a NC scheme defined over the complex field. Therefore, the PANC proposed above may be considered as a conventional NC scheme exhibiting a channel coding gain, which is a benefit of the mutual dependencies introduced by the linear module 2 addition of multiple streams.

\section{Iterative Detection and Decoding}

1) Receiver Structure: The destination receives $N$ CSs' transmitted signals $x_{n}^{(2)}, n \in[1, N]$ simultaneously, which experienced independently faded channels $h_{n}$, yielding a multiple accessed received signal. The receiver uses iterative data detection (DET) and channel decoding (DEC) as seen in Fig. 1. Both the SPC and PANC aided MSC may use the same DET algorithm. A host of DET schemes may be invoked, including the powerful but complex Maximum Likelihood (ML) detection scheme [9] or a low-complexity soft interference cancellation scheme [10]. The main difference between employing a PANC and a SPC scheme from a DEC point of view is that the PANC arrangement benefits from the joint decoding of multiple information streams, while SPC performs single-stream channel-decoding, as seen in Fig. 1. The soft channel decoder design of SPC aided MSC depends on the choice of the specific outer channel coding function. Hence here we discuss the soft decoding of PANC only.

2) Decoding of the PANC: The soft decoding of the PANC is analogous to that of a Repeat Accumulate (RA) code [11]. As seen in Fig. 1, it consists of the soft ACC decoder and soft combiner (COM). When considering a non-systematic PANC, after inputting the soft output information of the DET $\left(\mathcal{L}_{\text {det }}^{e}\right)$ to the DEC, the soft output of the ACC is forwarded to the soft combiner COM $\left(\mathcal{L}_{a c c}^{e}\right)$ of all $\kappa$ versions of the differently interleaved information streams $\left[\pi_{1}(\mathbf{s}), \ldots, \pi_{\kappa}(\mathbf{s})\right]$, which are then soft-combined and fed back to the ACC decoder $\left(\mathcal{L}_{\text {com }}^{e}\right)$ for the sake of providing updated soft-information for the DET $\left(\mathcal{L}_{\text {dec }}^{e}\right)$. When a systematic PANC is employed, the soft-output of the ACC decoder provided for the COM block of Fig. 1 corresponds to all $\kappa^{n s}=\left(\kappa-\kappa^{s}\right)$ versions of the differently interleaved 
TABLE I

SimUlation PARAMETERS IN Fig. 2 AND FIg. 3

\begin{tabular}{|c|c|c|c|c|c||c|c|}
\hline \multicolumn{2}{|l|}{} & \multicolumn{5}{|c||}{ SPC } & \multicolumn{2}{c|}{ PANC } \\
\hline \hline$\eta$ & $N$ & $R_{1}$ & $R_{2}$ & $L$ & $\iota$ & $R_{c}\left(\kappa^{s}, \kappa^{n s}\right)$ & $\iota$ \\
\hline $1 / 2$ & 2 & $1 / 4$ & $1 / 2$ & 2 & $(1,5) \times 20$ & $1 / 4(0,4)$ & 20 \\
\hline 1 & 4 & $1 / 4$ & $1 / 4$ & 4 & $(1,5,10) \times 30$ & $1 / 4(0,4)$ & 30 \\
\cline { 6 - 7 } & & & & & $1 / 4(1,3)$ & 30 \\
\hline
\end{tabular}

information streams $\left[\pi_{\kappa^{n s}+1}(\mathbf{s}), \ldots, \pi_{\kappa}(\mathbf{s})\right]$. The rest of the soft-information related to the $\kappa^{s}$ versions of the differently interleaved information streams $\left[\pi_{1}(\mathbf{s}), \pi_{2}(\mathbf{s}), \ldots, \pi_{\kappa^{s}}(\mathbf{s})\right]$ is directly fed to the soft-combiner block COM of Fig. 1, which means that there is no ACC decoding block between the DET and COM blocks. After carrying out all the affordable iterations, the soft COM block of Fig. 1 delivers its ultimate soft decision $\mathcal{L}_{\text {com }}^{p}$ concerning $\mathbf{s}_{i}, i \in[1, K]$. With the aid of factor graph representation of ACC and COM, the involved soft-information obeys the classic sum-product algorithm [12].

\section{Performance Evaluation}

\section{A. Assumptions and Parameters}

Let us now quantify the achievable performance of the proposed coding schemes. We assume error-free Phase-I cooperation, which is achieved by ensuring that cooperation is only activated by a perfect CRC check. The flat Rayleigh block fading channels $h_{n}, n \in[1, N]$ between the $N$ CSs and the destination are assumed to be independent and are perfectly known at the destination.

Before comparing these two coding schemes, we define our performance metric set $\chi$, which consists of the achievable throughput $\eta$, the block error ratio $P_{e}^{b l}$, the delay $\tau$ and the complexity $\iota$, i.e. we have $\chi=\left\{\eta, P_{e}^{b l}, \tau, \iota\right\}$. The system's effective throughput $\eta$ may be defined as $\eta=R_{c} N L$, where $R_{C}$ is the channel coding rate, $N$ is the number of CSs, $L$ is the number of layers when the SPC scheme is employed, while we have $L=1$ when the PANC scheme is considered. For the SPC scheme, because of this similarity between RA code and PANC, we employ a rate $R_{1}$ regular non-systematic RA code as the outer channel code in conjunction with a rate $R_{2}$ repetition code in order to facilitate the multiple layers' superposition. Thus the total code-rate becomes $R_{c}=R_{1} R_{2}$. On the other hand, the code-rate $R_{c}$ of the PANC is defined as the number of differently interleaved versions $\kappa$.

Therefore, by setting the same system throughput $\eta$ and the same source information segment length of $N_{i}=512$ symbols, resulting in a fixed delay $\tau$, we compare the two coding schemes in terms of their block error ratio $P_{e}^{b l}$ and associated complexity $\iota$. The complexity $\iota$ is simply quantified in terms of the number of iterations invoked. The total number of iterations of a SPC aided MSC scheme is the product of the number of DET $\rightleftharpoons$ DEC iterations and the number of iterations within the RA code, while that of a PANC aided system is deemed to be proportional to the number of iterations invoked by the three-stage DET $\rightleftharpoons$ $\mathrm{ACC} \rightleftharpoons \mathrm{COM}$ decoder chain. The simulation parameters used are summarized in Table I.

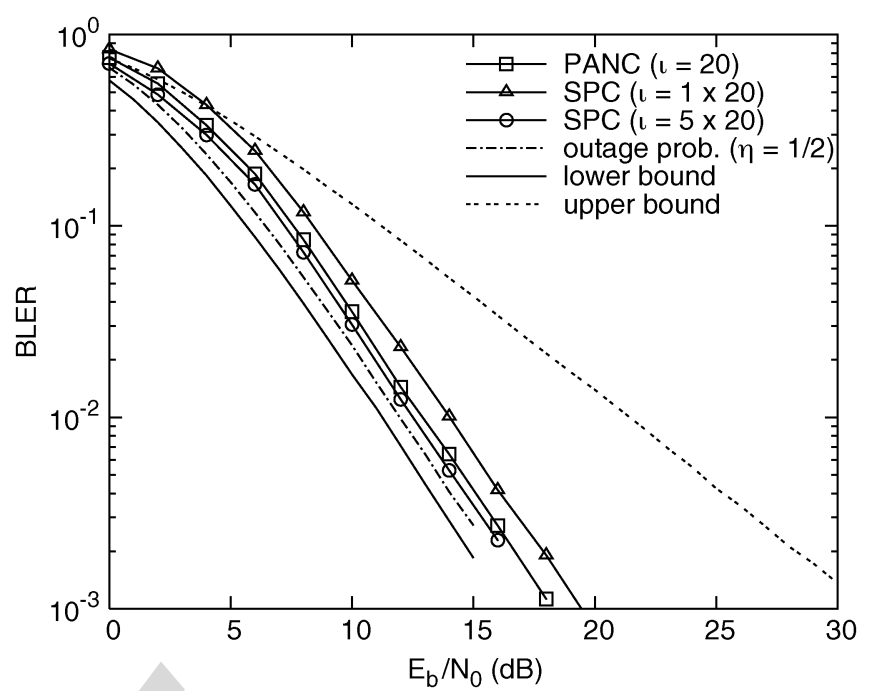

Fig. 2. BLER of the SPC and PANC scheme aided MSC against their upper and lower bounds, where we have $K=N=2$ and $\eta=1 / 2$. The simulation parameters are listed in Table I.

\section{B. Outage Bound Analysis}

We now perform an outage bound analysis as a reference for the cooperative coding schemes proposed in Section II. Without loss of generality, we discuss the $N=2 \mathrm{MSC}$ aided scenario. The maximum mutual information $I$ of an $N=2$ MSC-aided multiple access channel is equal to the minimum amongst the individual source's mutual information $I_{1}, I_{2}$ and the sum mutual information $I_{s}$, which is given by [13] $I=\min I_{1}\left(g_{1}\right), I_{2}\left(g_{2}\right), I_{s}\left(g_{s}\right)$, where $I_{i}\left(g_{i}\right)$, $i \in\{1,2, s\}$ is written in the classic form as a function of the Signal-to-Noise-Ratio (SNR) $\gamma_{s}$ and the effective channel gain $g_{i}$, more explicitly we have $I_{i}\left(g_{i}\right)=\log _{2}\left(1+g_{i} \gamma_{s}\right), i=\{1,2\}$ and $I_{s}\left(g_{s}\right)=(1 / 2) \log _{2}\left(1+g_{s} \gamma_{s}\right)$. For no cooperation scenario upper bound (u.b.), $g_{i}=\left|h_{i}\right|^{2}, i=\{1,2\}$, while for cooperation scenario lower bound (1.b.), $g_{i}=\left|h_{12}\right|+\left|h_{i}\right|^{2}, i=\{1,2\}$, where $\left|h_{12}\right|=\left|h_{21}\right|$ denotes the identical inter-source channels. In both scenarios, $g_{s}=\left(\left|h_{1}\right|^{2}+\left|h_{2}\right|^{2}\right) / 2$.

The outage probability of a fading channel is defined as the probability of having a mutual information between the received soft value and the decided symbol, which is less than the system's target effective throughput $\eta$, formulated as $p_{\text {out }}=\operatorname{Pr}[I<\eta]$. Finding the outage probability $p_{\text {out }}$ at the system's target effective throughput $\eta$ and a given SNR per-bit $\gamma_{b}$ is equivalent to finding $p_{\text {out }}=\operatorname{Pr}\left[g<g_{\eta}\right]$, where $g_{\eta}=\left(2^{\eta}-1\right) / \eta \gamma_{b}$ and $g=\min \left\{g_{1}, g_{2}, g_{s}\right\}$. The minimum outage probability $p_{\text {out }}^{\text {min }}$ at a given $\gamma_{b}$ value is achieved by letting $\eta \rightarrow 0$ and it is well known that $\lim _{\eta \rightarrow 0}\left(2^{\eta}-1\right) / \eta=\ln 2$.

\section{Simulation Results}

Fig. 2 and Fig. 3 suggest that both of our proposed coding schemes are capable of approaching the outage probability bound at their corresponding system throughput $\eta$. In Fig. 2, the non-systematic PANC scheme employing $\iota_{P A N C}=20$ iterations performs within a small fraction of a $\mathrm{dB}$ from the SPC scheme, which requires a total of $\iota_{S P C}=100$ iterations, hence the former results in a significantly lower complexity. The same trend was also confirmed in Fig. 3. However, since 


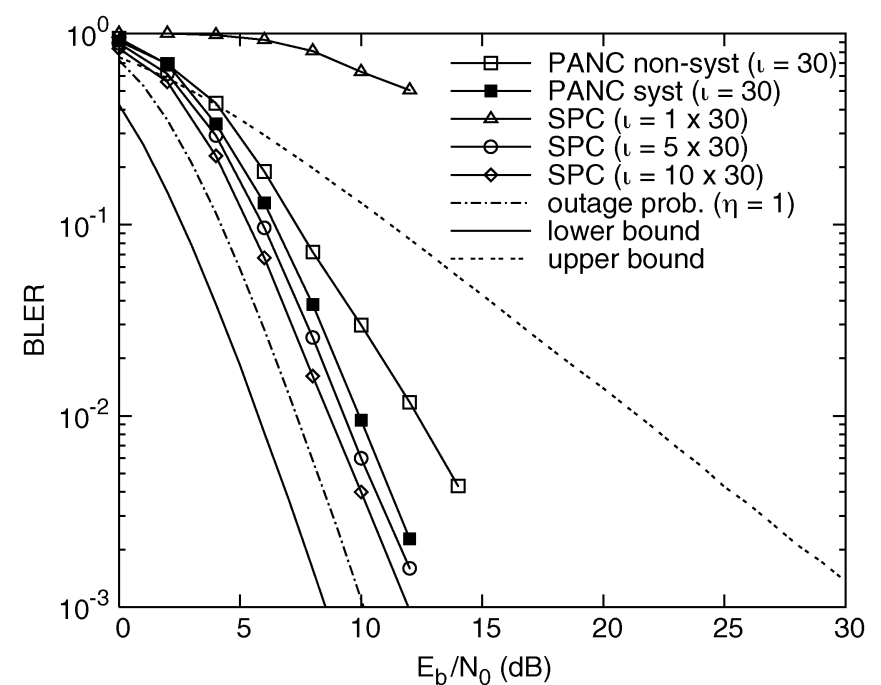

Fig. 3. BLER of the SPC and PANC scheme aided MSC against their upper and lower bounds, where we have $K=N=4$ and $\eta=1$. The simulation parameters are listed in Table I.

the effective system throughput $\eta$ was doubled from half to unity, both schemes exhibited a slightly higher discrepancy w.r.t. the outage probability bound. Futhermore, the systematic PANC performs better than its nonsystematic counterpart and its performance is close to that of the more complex SPC system. Since the complexity imposed determines the total power consumption, the PANC scheme may be considered as being more power-efficient.

It was found in Fig. 3 that the non-systematic PANC is unable to fully exploit the spatial transmit diversity gain provided by $N=4 \mathrm{CSs}$ due to its randomly designed nature. This is particularly true, when the effective throughput becomes unity, which may be referred to as a 'fully-loaded' MSC-aided scenario. The systematic PANC, on the other hand, provides an additional diversity gain, since the systematic information segment of a codeword facilitates direct communication with the soft COM of Fig. 1 at the cost of sacrificing some of the attainable coding gain. When the effective throughput is less than unity, i.e. the system is partially-loaded, the non-systematic PANC performs sufficiently well, as seen in Fig. 2. From a classic coding point of view [14], the systematic information segment provides a certain amount of direct a priori information for the soft COM of
Fig. 1, which becomes particularly crucial, when the a priori information gleaned from the ACC block of Fig. 1 is low, as in the fully-loaded scenario.

\section{CONCLUSION}

In this paper, we proposed two different coding schemes in the context of energy efficient MSC, namely the SPC and PANC schemes. The simulation results of Fig. 2 and Fig. 3 demonstrate that both schemes are capable of performing close to the outage probability bound. When compared to the SPC arrangement, the novel PANC scheme exhibits a lower complexity at the cost of a slight performance degradation, while maintaining the same effective throughput and delay.

\section{REFERENCES}

[1] O. Shalvi, "Multiple source cooperation diversity," IEEE Commun. Lett., vol. 8, pp. 712-714, Dec. 2004.

[2] A. Ribeiro, R. Wang, and G. Giannakis, "Multi-source cooperation with full-diversity spectral-efficiency and controllable-complexity," IEEE J. Sel. Areas Commun., vol. 25, pp. 415-425, Feb. 2007.

[3] R. Zhang and L. Hanzo, "Space-time coding for high-throughput interleave division multiplexing aided multi-source cooperation," Electron. Lett., vol. 28, pp. 367-368, Feb. 2008.

[4] R. Ahlswede, N. Cai, S.-Y. Li, and R. Yeung, "Network information flow," IEEE Trans. . Theory, vol. 4, pp. 1204-1216, July 2000.

[5] Y. Chen, S. Kishore, and J. Li, "Wireless diversity through network coding," in Proc. IEEE WCNC '06, Las Vegas, NV, Apr. 3-6, 2006, pp. $1681-1686$.

[6] C. Hausl and J. Hagenauer, "Iterative network and channel decoding for the two-way relay channel,' in Proc. IEEE ICC '06, Istanbul, Turkey, Jun. 11-15, 2006, pp. 1568-1573.

[7] L. Xiao, T. Fuja, J. Kliewer, and D. Costello, "A network coding approach to cooperative diversity," IEEE Trans. Inform. Theory, vol. 53, pp. 3714-3722, Oct. 2007.

[8] T. Wang and G. Giannakis, "Complex field network coding for multiuser cooperative communications," IEEE J. Sel. Areas Commun., vol. 26, pp. 561-571, Apr. 2008.

[9] L. Hanzo and T. Keller, OFDM and MC-CDMA: A Primer. Hoboken, NJ: Wiley-IEEE Press, 2006.

[10] K. Wu and L. Ping, "Multilayer turbo space-time codes," IEEE Trans. Commun., vol. 9, pp. 55-57, Jan. 2005.

[11] J. Jin, A. Khandekar, and R. J. McEliece, "Irregular repeat-accumulate codes," in Proc. 2nd Int. Conf. Turbo Codes, Munich, Germany, Sept. 4-7, 2000, pp. 125-127.

[12] F. Kschischang, B. Frey, and H. Loeliger, "Factor graphs and the sumproduct algorithm," IEEE Trans. Inform. Theory, vol. 47, pp. 498-519, Feb. 2001.

[13] T. Cover and J. Thomas, Elements of Information Theory. New York: Wiley, 1991.

[14] S. ten Brink and G. Kramer, "Design of repeat-accumulate codes for iterative detection and decoding," IEEE Trans. Signal Process., vol. 51, pp. 2764-2772, Nov. 2003. 